\title{
High thermoelectric figure of merit of quantum dot array quantum wires
}

\author{
David M T Kuo \\ Department of Electrical Engineering and Department of Physics, \\ National Central University, Chungli, 320 Taiwan
}

(Dated: June 3, 2021)

\begin{abstract}
How to design silicon-based quantum wires with figure of merit $(Z T)$ larger than three is under hot pursuit due to the advantage of low cost and the availability of matured fabrication technique. Quantum wires consisting of finite three dimensional quantum dot (QD) arrays coupled to electrodes are proposed to realize high efficient thermoelectric devices with optimized power factors. The transmission coefficient of 3D QD arrays can exhibit 3D, 2D, 1D and 0D topological distribution functions by tailoring the interdot coupling strengths. Such topological effects on the thermoelectric properties are revealed. The 1D topological distribution function shows the maximum power factor and the best $Z T$ value. We have demonstrated that $3 \mathrm{D}$ silicon $\mathrm{QD}$ array nanowires with diameters below $20 \mathrm{~nm}$ and length $250 \mathrm{~nm}$ show high potential to achieve $Z T \geq 3$ near room temperature.
\end{abstract}

\section{INTRODUCTION}

Thermoelectric power is one of the most important green energies, which play a remarkable role for eternal development of earth.[1] Unlike solar cells and windpower generators, thermoelectric devices have not only the functionality of power generators but also coolers. Designing a thermoelectric material with a high figure of merit $(Z T)$ and optimized power output is under pursuit. [2-5] Extensive studies have shown that it is difficult to achieve a $Z T$ larger than one at room temperature in bulk thermoelectric materials.[1] With the advances of semiconductor technology, many experiments nowadays can realize $Z T$ larger than one at room temperature in low-dimensional systems.[6,7] If a material with $Z T \geq 3$ at room temperature it will tremendously brighten the scenario of thermoelectric devices[1]. The enhancement of power output call for a large number of electronic states (bulk-like). However, a large $Z T$ value occurs in dilute electronic states and phonon excitations (atom-like).[8]

It has been predicted theoretically that $Z T \geq 3$ can be achieved in thin semiconductor nanowires with diameters smaller than $3 \mathrm{~nm}[9,10]$. However, no experimental realization of such impressive thermoelectric devices has been reported[1]. The finding of $Z T=1$ in silicon nanowires at room temperature[11] has inspired further studies of thermoelectric properties of siliconbased nanowires because of the advantages of low cost and the availability of matured fabrication technology in silicon industry[11-14]. Whether $Z T \geq 3$ exists in siliconbased nanowires at room temperature becomes an interesting topic.[14] It is calculated that Si/Ge quantum dot suerlattice (QDSL) nanowire with an optimized period around $5 \mathrm{~nm}$ and cross-sectional area around $3 \mathrm{~nm} \times 3 \mathrm{~nm}$ can lead to an reduction of phonon thermal conductance by one order of magnitude in comparison with pristine Si nanowires $[15,16]$. Therefore, it is desirable to study the power output and $Z T$ of quantum wire consisting of finite 3D QD arrays (QDAs) in ballistic transport procedure as depicted in Fig. 1. Under tailoring interdot coupling strengths, the transmission coefficient of finite size 3D QDAs can exhibit 3D, 2D, 1D and 0D distribution functions. Their effects on the thermoelectric properties of finite size QDA nanowires are examined. We found that $Z T \geq 3$ at room temperature may be achieved in a quantum wire made of finite $3 \mathrm{D} \mathrm{Si} / \mathrm{Ge}$ QDAs with a diameter below $20 \mathrm{~nm}$ and a length $L_{x}=250 \mathrm{~nm}$.

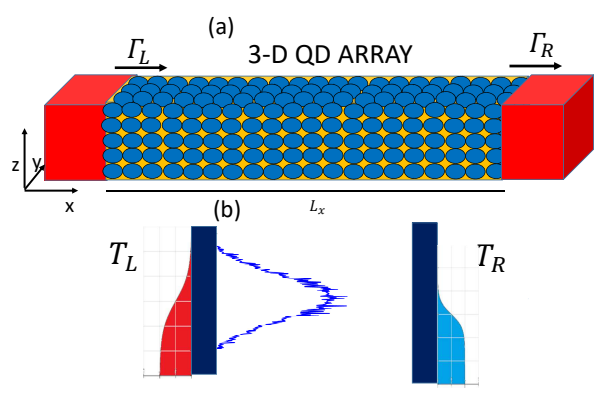

FIG. 1: (a) Schematic diagram of a quantum wire consisting of finite 3D quantum dot (QD) array coupled to electrodes. $\Gamma_{L}\left(\Gamma_{R}\right)$ denotes the tunneling rate of the electrons between the left (right) electrode and the leftmost (rightmost) QDs. (b) Electronic spectra of a 3D QD array coupled to electrodes with different equilibrium temperatures $\left(T_{L}\right.$ and $\left.T_{R}\right)$.

\section{FORMALISM}

To model the thermoelectric properties of a finite size QDA nanowire connected to the electrodes, the Hamiltonian of the system shown in Fig. 1 is given by $H=$ $H_{0}+H_{Q D},[17]$ where

$$
\begin{aligned}
H_{0} & =\sum_{k, \sigma} \epsilon_{k} a_{k, \sigma}^{\dagger} a_{k, \sigma}+\sum_{k, \sigma} \epsilon_{k} b_{k, \sigma}^{\dagger} b_{k, \sigma} \\
& +\sum_{\ell} \sum_{k, \sigma} V_{k, \ell}^{L} d_{\ell, \sigma}^{\dagger} a_{k, \sigma}+\sum_{\ell} \sum_{k, \sigma} V_{k, \ell}^{R} d_{\ell, \sigma}^{\dagger} b_{k, \sigma}+H . c .
\end{aligned}
$$

The first two terms of Eq. (1) describe the free electron gas in the left and right electrodes. $a_{k, \sigma}^{\dagger}\left(b_{k, \sigma}^{\dagger}\right)$ creates an electron of momentum $k$ and spin $\sigma$ with energy $\epsilon_{k}$ in the 
left (right) electrode. $V_{k, \ell}^{L}\left(V_{k, \ell}^{R}\right)$ describes the coupling between the left (right) lead with its adjacent QD in the th site.

$$
H_{Q D}=\sum_{\ell, \sigma} E_{0} d_{\ell, \sigma}^{\dagger} d_{\ell, \sigma}+\sum_{\ell, j, \sigma}-t_{\ell, j} d_{\ell, \sigma}^{\dagger} d_{j, \sigma}+H . c,(2)
$$

where $E_{0}$ is the site-independent energy level of QD. The spin-independent $t_{\ell, j}$ describes the interdot coupling strength, which is limited to the nearest neighboring sites. $d_{\ell, \sigma}^{\dagger}\left(d_{\ell, \sigma}\right)$ creates (destroys) one electron in the $\ell$ th site QD. If the wave functions of the electrons in each QD are localized, the electron Coulomb interactions are strong. Their effects on electron transport are significant in the scenario of weak interdot coupling strengths.[18] On the other hand, the wave functions of the electrons are delocalized in the scenario of strong interdot coupling strengths to form minibands; hence their weak electron Coulomb interactions can be ignored. In eqs. (1) and (2), we take into account one energy level for each QD due to nanoscale QDs considered.

To study the transport properties of a QDA nanowire junction connected to electrodes, it is convenient to use the Keldysh-Green's function technique[17]. Electron and heat currents leaving electrodes can be expressed as

$$
J=\frac{\sigma_{s} e}{h} \int d \epsilon T_{L R}(\epsilon)\left[f_{L}(\epsilon)-f_{R}(\epsilon)\right]
$$

and

$$
\begin{aligned}
& Q_{e, L(R)} \\
= & \frac{ \pm \sigma_{s}}{h} \int d \epsilon T_{L R}(\epsilon)\left(\epsilon-\mu_{L(R)}\right)\left[f_{L}(\epsilon)-f_{R}(\epsilon)\right]
\end{aligned}
$$

where $f_{\alpha}(\epsilon)=1 /\left\{\exp \left[\left(\epsilon-\mu_{\alpha}\right) / k_{B} T_{\alpha}\right]+1\right\}$ denotes the Fermi distribution function for the $\alpha$-th electrode, where $\mu_{\alpha}$ and $T_{\alpha}$ are the chemical potential and the temperature of the $\alpha$ electrode. $e, h$, and $k_{B}$ denote the electron charge, the Planck's constant, and the Boltzmann constant, in that order. The factor $\sigma_{s}$ include electron spin and valley degeneracy of QDs. $T_{L R}(\epsilon)$ denotes the transmission coefficient of a 3D QDA connected to electrodes, which can be solved by the formula $T_{L R}(\epsilon)=4 \operatorname{Tr}\left[\hat{\Gamma}_{L} \hat{G}_{Q D A}^{r}(\epsilon) \hat{\Gamma}_{R} \hat{G}_{Q D A}^{a}(\epsilon)\right],[19-21]$ where the matrix of tunneling rates $\left(\hat{\Gamma}_{L}\right.$ and $\left.\hat{\Gamma}_{R}\right)$ and Green's functions $\left(\hat{G}_{Q D A}^{r}(\epsilon)\right.$ and $\left.\hat{G}_{Q D A}^{a}(\epsilon)\right)$ can be constructed by coding.[21]

The electrical conductance $\left(G_{e}\right)$, Seebeck coefficient $(S)$ and electron thermal conductance $\left(\kappa_{e}\right)$ can be evaluated by using Eqs. (3) and (4) with a small applied bias $\Delta V=\left(\mu_{L}-\mu_{R}\right) / e$ and cross-junction temperature difference $\Delta T=T_{L}-T_{R}$. We obtain these thermoelectric coefficients $G_{e}=e^{2} \mathcal{L}_{0}, S=-\mathcal{L}_{1} /\left(e T \mathcal{L}_{0}\right)$ and $\kappa_{e}=\frac{1}{T}\left(\mathcal{L}_{2}-\mathcal{L}_{1}^{2} / \mathcal{L}_{0}\right) . \mathcal{L}_{n}$ is given by

$$
\mathcal{L}_{n}=\frac{\sigma_{s}}{h} \int d \epsilon T_{L R}(\epsilon)(\epsilon-\mu)^{n} \frac{-\partial f(\epsilon)}{\partial \epsilon},
$$

where $f(\epsilon)=1 /\left(\exp ^{(\epsilon-\mu) / k_{B} T}+1\right)$ is the Fermi distribution function of electrodes at equilibrium temperature $T$ and chemical potential $\mu$. Factor $\sigma_{s}=12$ is for silicon QDs.

\section{RESULTS AND DISCUSSION}

To illustrate the thermoelectric properties of finite 3D QDAs, we have calculated and shown in Fig. 2 transmission coefficient $T_{L R}(\epsilon)$ as a function of $\epsilon$ for different interdot coupling strength configurations at tunneling rate $\Gamma_{t}=6 \Gamma_{0}\left(\Gamma_{L(R), \ell}(\epsilon)=2 \pi \sum_{k}\left|V_{k, \ell}^{L(R)}\right|^{2} \delta\left(\epsilon-\epsilon_{k}\right)=\Gamma_{t}\right)$ and QDAs with $N_{x}=25$ and $N_{y}=N_{z}=N_{r}=5$. We employ $t_{x}, t_{y}$ and $t_{z}$ to illustrate the interdot coupling strength $t_{\ell, j}$ in the $\mathrm{x}, \mathrm{y}$ and $\mathrm{z}$ directions, respectively. Diagram (a) considers homogenous interdot coupling strengths $t_{x}=t_{y}=t_{z}=6 \Gamma_{0}$ to describe finite $3 \mathrm{D}$ QDAs. We turn off $t_{z}=0$ in diagram (b) to illustrate multi-layer 2D structures. In diagram (c), $t_{z}=t_{y}=0$ describes multi 1D QDSLs. All energy scales of physical parameters are in units of $\Gamma_{0}=1 \mathrm{meV}$. Fig. 2 reveals the topological effect on $T_{L R}(\epsilon)$ and the tunneling probability of the electrons of the electrodes through the electronic states of QDAs. Those electronic states are described by $\epsilon=E_{0}-\left(2 t_{x} \cos \left(\frac{n_{x} \pi}{N_{x}+1}\right)+2 t_{y} \cos \left(\frac{n_{y} \pi}{N_{y}+1}\right)+2 t_{z} \cos \left(\frac{n_{z} \pi}{N_{z}+1}\right)\right)$, where $n_{x}=1,2, . . N_{x}, n_{y}=1,2, . . N_{y}$ and $n_{z}=1,2, . . N_{z}$. For the situation of $t_{x}=t_{y}=t_{z}=6 \Gamma_{0}$, a finite 3D QDA shows a 3D topological distribution function in Fig. 2(a). When $t_{z}=0$, we see a 2D topological distribution function in Fig. 2(b). We have a typical 1D topological distribution function for $t_{y}=t_{z}=0$, as seen in Fig. 2(c).[21] These electronic states have inhomogeneous broadening due to the leftmost and rightmost QDs of 3D QDAs connected to the electrodes. Electronic states near $E_{0}$ are more broadening than those near band edges. For 1D topological situation, lower band edge (LBE) and upper band edge (UBE) are, respectively, $-12 \Gamma_{0}$ and $12 \Gamma_{0}$. The area between LBE and UBE is so called the band regime (BR). For 2D and 3D topological situations, their band widths (BWs) are 48 and $72 \Gamma_{0}$, respectively. Physical parameters considered such as QD energy level $E_{0}$, inter-dot coupling strength and tunneling rate can be evaluated in the framework of effective mass model.[22] According to eqs. (3) and (4), the maximum electron current and heat current occur at $T_{L R}(\epsilon)$ with the maximum area at a fixed BW. Our previous work has demonstrated that the condition of $\Gamma_{t}=t_{x}$ has the optimization of $T_{L R}(\epsilon)$ of QDAs.[21] Therefore, $\Gamma_{t}=t_{x}$ will be used through out this article.

Fig. 3 shows the calculated $G_{e}, S$ and power factor $\left(P F=S^{2} G_{e}\right)$ at functions of $\mu$ for different topological distribution functions shown in Fig.2 at two different temperatures. Due to temperature effect, the highly oscillatory electronic states shown in $T_{L R}(\varepsilon)$ are washed out in the spectra of $G_{e}$. The maximum power factor in Fig. 3(c) is given by the $1 \mathrm{D}$ topological case. The $1 \mathrm{D}$ maximum $P F$ values occur at $|\mu|=14 \Gamma_{0}$, where the $1 \mathrm{D}$ 


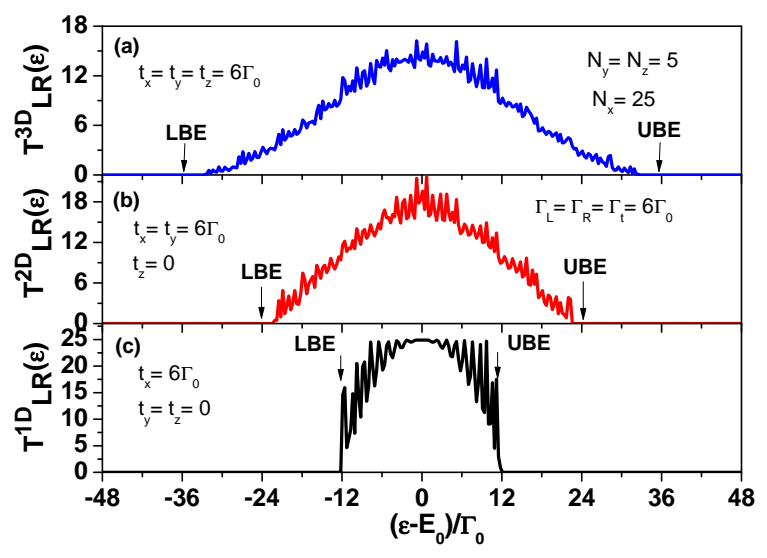

FIG. 2: Transmission coefficient $T_{L R}(\varepsilon)$ as functions of $\varepsilon$ for different inter-dot coupling strength configurations at $\Gamma_{L}=$ $\Gamma_{R}=\Gamma_{t}=6 \Gamma_{0}$ and $N_{x}=25, N_{y}=N_{z}=N_{r}=5$. We set $E_{0}=0$ through out this article.

Seebeck coefficient is significantly larger than that of 2D or 3D as seen in Fig 3(b). This is because 1D transmission coefficient shows the steep change with respect to $\epsilon$. In Fig. 3(f) the power factor is almost independent on the topological situations as $k_{B} T=25 \Gamma_{0}$. Based on eq. (5), the range of integration increases with increasing temperature. At $B W /\left(2 k_{B} T\right) \leq 1$ we have $\int d \varepsilon T_{L R}^{n D}(\varepsilon)=C$ where $C$ is a constant, this explains why the curves of $G_{e}$ and $S$ merge together at high temperatures. In particular, $S \approx \mu /(e T)$ in Fig. 3(e). To further clarify the behaviors of Fig. 3 (d) and (e), we assume transmission coefficient $T_{L R}(\epsilon)=4 \Gamma_{t}^{2} N_{r}^{2} /\left(\left(\epsilon-E_{0}\right)^{2}+\left(2 \Gamma_{t}\right)^{2}\right)$ and $\Gamma_{t} /\left(2 k_{B} T\right) \ll 1$ in eq. (5). $G_{e}=\frac{e^{2}}{h} \frac{N_{r}^{2} \pi \Gamma_{t}}{2 k_{B} T \cosh ^{2}\left(\mu /\left(2 k_{B} T\right)\right)}$ and $S=\mu /(e T)$ are obtained. The results of Fig. 3(e) indicate that the thermoelectric behavior of finite 3D QDAs with finite BWs is similar to that of a single QD coupled to electrodes as $B W /\left(2 k_{B} T\right) \leq 1$. In Fig. $3, G_{e}$ and $S$ do not change at $k_{B} T=25 \Gamma_{0}$ if we continuously increase $N_{x}$ at a fixed $N_{r}=5$ (see red curves in Fig.6).

To further clarify the behavior of thermoelectric coefficients with respect to temperature shown in Fig.3, we have calculated $G_{e}, S, P F$ and $Z T$ as functions of $k_{B} T$ at $\mu=-60 \Gamma_{0}$ and $N_{x}=50$ in Fig. 4. In addition, we have added extra curves to consider the $1 \mathrm{D}$ case with a smaller $t_{x}=\Gamma_{t}=2 \Gamma_{0}$ value, which is called atomic-limit $(0 D)$ to distinguish from the $1 \mathrm{D}$ case of $t_{x}=\Gamma_{t}=6 \Gamma_{0}$. Because only electronic states around $\mu$ give the contributions of electron transport between the electrodes at low temperatures, we see the vanishingly small conductance at $k_{B} T \leq 5 \Gamma_{0}$ for $\mu=-60 \Gamma_{0}$, which is far away from the band center $\left(E_{0}\right)$. Electron transport is mainly contributed to thermionic assisted tunneling process (TATP) as seen in Fig. 4(a). Due to the widest band width, $G_{e}$ shows the maximum conductance in the $3 \mathrm{D}$ case. As for Seebeck coefficients, it is expected that $S_{0 D}=\mu /(e T)$ has a maximum value. We note that $S_{0 D}$
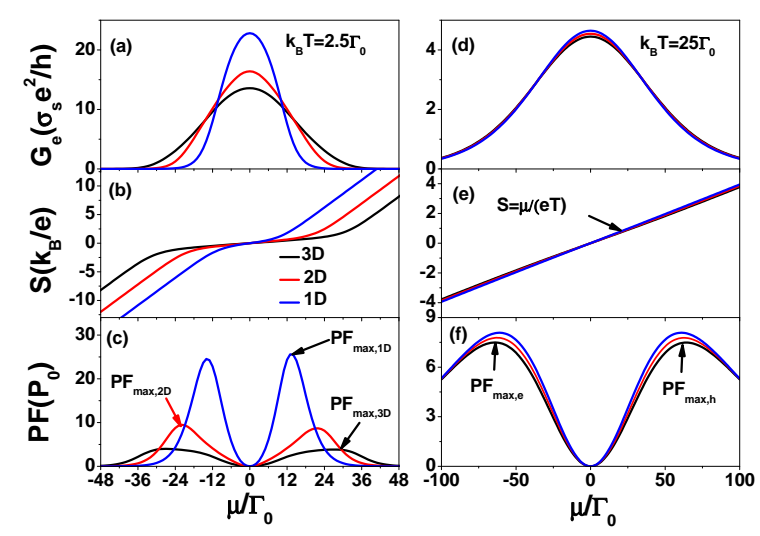

FIG. 3: (a) Electrical conductance $G_{e}$, (b) Seebeck coefficient $S$ and (c) power factor $P F=S^{2} G_{e}$ as functions of $\mu$ for three different topological spectra shown in Fig. 2 at $k_{B} T=2.5 \Gamma_{0}$. The curves of diagrams (d), (e) and (f) consider the case of $k_{B} T=25 \Gamma_{0}$. We have $P_{0}=\sigma_{s} k_{B}^{2} / h^{2}$.

is determined only by $T$ as $\mu$ is fixed. In particular, the dimensional effect on $S$ is disappear at $k_{B} T \geq 25 \Gamma_{0}$. This unique characteristic of $S$ may exist a useful application of temperature sensors. When $k_{B} T>10 \Gamma_{0}$, the trend of $P F_{1 D}>P F_{2 D}>P F_{3 D}$ is seen in Fig. 4(c). The maximum power factor reaches $P F_{1 D, \max }=8 P_{0}$ at room temperature. This indicates that system provides the electrical power output $7.82 \mathrm{nW} / \mathrm{K}$ at room temperature. Although $S_{0 D}$ has the largest value in the regime of $k_{B} T>5 \Gamma_{0}$, its power factor is poor.
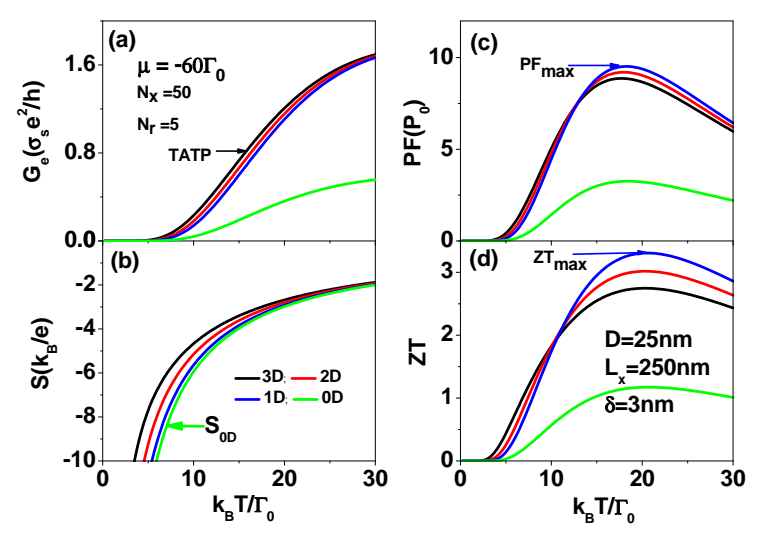

FIG. 4: (a) Electrical conductance, (b) Seeback coefficient, (c) power factor $P F$ and (d) figure of merit $Z T$ as functions of $k_{B} T$ at $\mu=-60 \Gamma_{0}, N_{r}=5$ and $N_{x}=50$. To calculate $\kappa_{p h}$, we have adopted diameter $D=25 \mathrm{~nm}$, length $L_{x}=250 \mathrm{~nm}$ and surface roughness $\delta=3 \mathrm{~nm}$.

The thermoelectric efficiency of materials is determined by the figure of merit, $Z T=S^{2} G_{e} T /\left(\kappa_{e}+\kappa_{p h}\right)$. $\kappa_{p h}$ denotes the phonon thermal conductance of finite $3 \mathrm{D}$ QDAs. It is difficult to fully block phonon heat currents 
because QDs are embedded into matrix.[18] We should include $\kappa_{p h}$ in the calculation of $Z T$. We have adopted the following empirical formula given by Ref.[23]

$$
\kappa_{p h}=\frac{F_{s}}{h} \int d \omega \mathcal{T}_{p h}(\omega) \frac{\hbar^{3} \omega^{2}}{k_{B} T^{2}} \frac{e^{\hbar \omega / k_{B} T}}{\left(e^{\hbar \omega / k_{B} T}-1\right)^{2}},
$$

where $\omega$ and $\mathcal{T}_{p h}(\omega)$ are the phonon frequency and throughput function, respectively. A dimensionless factor $F_{s}$ is introduced to describe the reduction factor for phonon transport due to scattering from QDs embedded in a quantum wire. The value of $F_{s}=0.1$ is used, which is determined according to Ref.[16], in which the phonon thermal conductance of silicon/germanium QD nanowires is calculated. As $F_{s}=1$, Eq. (6) well explains the $\kappa_{p h}$ of silicon quantum wires with diameters $D \geq 20 \mathrm{~nm} .[23,24]$. In Fig. 4(d) we have considered a quantum wire with diameter $D=25 \mathrm{~nm}$ and length $L_{x}=250 \mathrm{~nm}$ since the pair length (one QD plus spacer layer) adopted is $L_{s}=5 \mathrm{~nm}$. [16] The maximum $Z T$ is given by $(Z T)_{1 D}=3.31$ at $k_{B} T=20 \Gamma_{0}$. This is a remarkable result, since quantum wires with $Z T \geq 3$ have been theoretically reported only for $D<3 \mathrm{~nm}$. [1,9] So far, we are limited to the situation of $N_{z}=N_{y}=N_{r}=5$. Although the power factor can be enhanced with increasing $N_{r}, \kappa_{p h}$ is also highly enhanced with increasing $D$.[23] We show the calculated $\kappa_{p h}$ as functions of temperature for different diameters at $L_{x}=250 \mathrm{~nm}$ in Fig. 5. The line marked with triangles denotes the $\kappa_{e}$ corresponding to the curves of $3 \mathrm{D}$ case in Fig. $4 . \kappa_{p h}$ fully dominates heat current at room temperature $\left(k_{B} T=25 \Gamma_{0}\right)$. It is worthy noting that $\kappa_{e}>\kappa_{p h}$ is observed in the situation of extremely low temperatures $k_{B} T<0.1 \Gamma_{0}$. Many literatures have reported the Carnot engine behavior of a single QD when $\kappa_{e} \gg \kappa_{p h} \cdot[25-27]$.

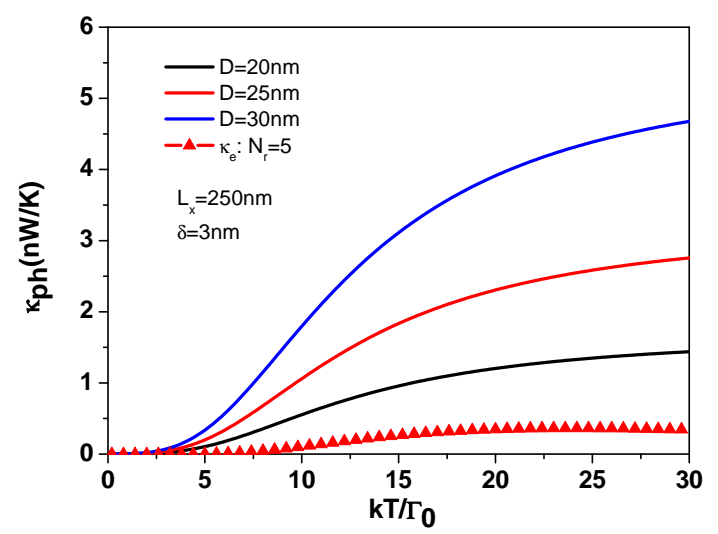

FIG. 5: Phonon thermal conductance $\kappa_{p h}$ as functions of temperature for different diameters at length $L_{x}=250 \mathrm{~nm}$ and surface roughness $\delta=3 \mathrm{~nm}$.

It is important to investigate the effect of quantum wire diameter on $Z T$ because $\kappa_{p h}$ is a function of $D$. We show the calculated $G_{e}, S, P F$ and $Z T$ as functions of $\mu$ for different $N_{r}$ values at $k_{B} T=25 \Gamma_{0}, N_{x}=50$ and $t_{x}=t_{y}=t_{z}=\Gamma_{t}=6 \Gamma_{0}$ in Fig. 6. From experimental point of view, it is relatively easy to fabricate the case of $t_{x}=t_{y}=t_{z} \cdot[28,29]$ For comparison, $0 D$ case is also included in Fig. 6. $G_{e}$ is highly enhanced with increasing $N_{r}$, whereas the enhancement of $G_{e}$ does not suppress $S$. As a consequence, $P F$ is enhanced with increasing $N_{r}$. This crucial characteristic is meaningful to increase the electrical power output. Quantum wire diameters are, respectively, $D=20,25$ and $30 \mathrm{~nm}$ for $N_{r}=4,5$ and 6 . In Fig. $6(\mathrm{~d})$ the maximum $Z T=3.31$ is given by $D=20 \mathrm{~nm}$ and $\mu=-70 \Gamma_{0}$. Note that the curve of a QDA nanowire exhibiting a $0 \mathrm{D}$ topological electron distribution function exists poor $P F$ and $Z T$ values when phonon throughput function maintains a $3 \mathrm{D}$ distribution function characteristic. In Ref.[2], $T_{L R}^{0 D}(\epsilon)$ shows the Carnot efficiency because they assumed that the condition of $\kappa_{e} \gg \kappa_{p h}$ can be realized experimentally.
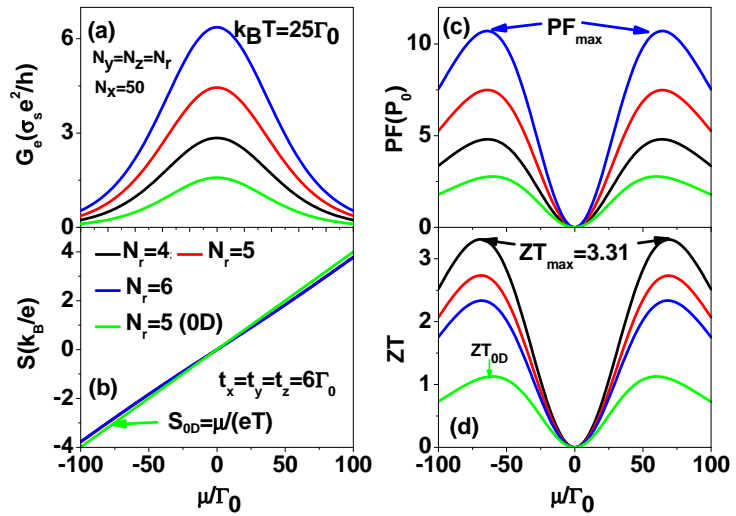

FIG. 6: (a) Electrical conductance, (b) Seeback coefficient, (c) power factor $P F$ and (d) figure of merit $Z T$ as functions of $\mu$ for various $N_{r}$ values at $t_{x}=t_{y}=t_{z}=6 \Gamma_{0}$. In the calculation of $\kappa_{p h}$, we have adopted $D=20,25$ and $30 \mathrm{~nm}$ for $N_{r}=4,5$ and 6 , respectively. The curve of $0 D$ corresponds to that of Fig. 4. Other physical parameters are the same as those of Fig.4.

\section{CONCLUSION}

We have theoretically investigated the thermoelectric properties of QDA quantum wires in the ballistic transport regime. According to Ref.[30], the electron mean free path $\lambda_{e}$ reaches $300 \mathrm{~nm}$ at room temperature in $\mathrm{Si}$ nanowires. Therefore, it is adequate to consider electron transport in the ballistic regime at $L_{x} \leq 250 \mathrm{~nm}$. The interdot coupling strengths $t_{\ell, j}$ are determined by the barrier width and height, which can be designed on the demand. For example, if $\mathrm{Si}$ QDs have $\mathrm{SiO}_{2}$ barrier widths, $t_{\ell, j}$ are vanishingly small. Tailoring $t_{\ell, j}, T_{L R}(\epsilon)$ exhibits the $3 \mathrm{D}, 2 \mathrm{D}, 1 \mathrm{D}$ and $0 \mathrm{D}$ topological distribution func- 
tions. We have revealed such topological effects on the power factor and figure of merit of quantum wires. Although $T_{L R}^{1 D}(\epsilon)$ provides the maximum power factor and the largest $Z T, T_{L R}^{3 D}(\epsilon)$ still shows a $Z T>3$ for a finite size silicon QDA nanowire with a diameter $D=20 \mathrm{~nm}$ and a length $L_{x}=250 \mathrm{~nm}$ in the TATP.

\section{Acknowledgments}

This work was supported under Contract No. MOST
110-2119-M-008-006-MBK

E-mail address: mtkuo@ee.ncu.edu.tw
1 A. J. Minnich, M. S. Dresselhaus, Z. F. Ren and G. Chen, Energy Environ. Sci 2, 466 (2009).

2 G. D. Mahan, and L. M. Woods, Phys. Rev. Lett. 80, 4016 (1998).

3 G. Chen, M. S. Dresselhaus, G. Dresselhaus, J. P. Fleurial, and T. Caillat, International Materials Reviews, 48, 45 (2003).

4 David. M.-T. Kuo and Y. C. Chang, Phys. Rev. B 81, 205321 (2010).

${ }^{5}$ R. S. Whitney, Phys. Rev. Lett. 112, 130601 (2014).

${ }^{6}$ R. Venkatasubramanian, E. Siivola, T. T. Colpitts and B. Q. Quinn, Nature 413, 597 (2001).

7) T. C. Harman, P. J. Taylor, M. P. Walsh and L. B. LaForge, Science 297, 2229 (2002).

8 G. D. Mahan and J. O. Sofo, Proc. Natl. Acad. Sci. USA 93, 7436 (1996).

9 L. D. Hicks, and M. S. Dresselhaus, Phys. Rev. B 47, 16631 (1993).

10 N. Mingo, Appl. Phys. Lett. 84, 2652 (2004).

11 A. I. Boukai, Y. Bunimovich, J. Tahir-Kheli, J. K. Yu, G. W. A, and J. R. Heath, Nature 415, 168 (2008).

12 D. Grutzmacher et al, Nano Lett. 7, 3150 (2007).

13 A. I. Hochbaum, R. K. Chen, R. D. Delgado, W. J. Liang, E. C. Garnett, M. Najarian, A. Majumdar,and P. D. Yang, Nature 451, 165 (2008).

14 J. H. Lee, J. W. Lim, and P. D. Yan, Nano Lett. 15, 3273 (2015).

15 D. L. Nika, E. P. Pokatilov, A. A. Balandin, V. M. Fomin, A. Rastelli, and O. G. Schmidt, Phys. Rev. B 84, 165415 (2011).
16 M. Hu and D. Poulikakos, Nano Lett. 12, 5487 (2012).

17 H. Haug and A. P. Jauho, Quantum Kinetics in Transport and Optics of Semiconductors (Springer, Heidelberg, 1996).

18 David. M. T. Kuo, C. C. Chen and Y. C. Chang, Phys. Rev. B 95, 075432 (2017).

19 Y. F. Zhou, H. Jiang, X. C. Xie and Q. F. Sun, Phys. Rev. B 95, 245137 (2017).

20 N. X. Yang, Y. F. Zhou, P. Lv and Q. F. Sun, Phys. Rev. B 97, 235435 (2018).

21 David. M. T. Kuo, AIP Advances 10, 045222 (2020).

22 David M.-T. Kuo and Y. C. Chang, Phys. Rev. B 61, 11051 (2000)

23 R. K. Chen, A. I. Hochbaum, P. Murphy, J. Moore, P. D. Yang, and A. Majumdar, Phys. Rev. Lett. 101, 105501 (2008).

24 D. Li, Y. Y. Wu, P. Kim, L. Shi, P. D. Yang, and A. Majumdar, Appl. Phys. Lett. 83, 2934 (2003).

25 P. Murphy, S. Mukerjee, and J. Moore, Phys. Rev. B 78 , 161406(R) (2008).

26 J. Liu, Q. F. Sun, and X. C. Xie, Phys. Rev. B 81, 245323 (2010).

27 P. Trocha and J. Barnas, Phys. Rev. B 85, 085408 (2012).

28 G. L. Chen, D. M. T Kuo, W. T. Lai and P. W. Li, Nanotechnology 18, 475402 (2007).

29 C. R. Kagan and C. B. Murry, Nature Nanotechnology 10, 1013 (2015).

30 W. Lu, J. Xiang, B. P. Timko, Y. Wu, and C. M. Lieber, Proc. Natl. Acad. Sci. U.S. A. 102, 10046 (2005). 